\title{
Effet des dates de semis sur la performance agronomique de Lagenaria siceraria (Molina) Standl. (Cucurbitaceae) et Manihot esculenta Crantz (Euphorbiaceae) en culture associée
}

\author{
Irié R. Djè Bi ${ }^{(1)}$, Kouadio I. Kouassi ${ }^{(1)}$, Kouamé K. Koffi ${ }^{(1)}$, Kouakou L. Kouakou ${ }^{(1)}$, \\ Jean-Pierre Baudoin ${ }^{(2)}$, Bi Irié A. Zoro ${ }^{(1)}$ \\ (1) Université Nangui Abrogoua. UFR des Sciences de la Nature. Unité de Phytotechnie et Amélioration Génétique (UPAG). \\ 02 BP 801 Abidjan 02 (Côte d'Ivoire).E-mail : kouadioignace@yahoo.fr, kouassignace_sn@una.edu.ci \\ (2) Université de Liège - Gembloux Agro-Bio Tech. Unité de Phytotechnie tropicale et d'Horticulture. Passage des Déportés, \\ 2. BE-5030 Gembloux (Belgique).
}

Reçu le 30 avril 2016, accepté le 22 mai 2017, mis en ligne le 8 aout 2017.

Cet article est distribué suivant les termes et les conditions de la licence CC-BY (http://creativecommons.org/licenses/by/4.0/ deed.fr)

Description du sujet. L'association culturale est une pratique bénéfique largement utilisée dans les zones tropicales pour la gestion durable de la fertilité des sols, l'amélioration des rendements de l'agriculture traditionnelle et des revenus des petits producteurs.

Objectifs. Évaluer l'effet de la culture associée et de la culture pure de la cucurbite Lagenaria siceraria, plante lianescente et du manioc Manihot esculenta var. 'Bonoua blanc', plante érigée, sur les différentes performances agronomiques de chacune de ces cultures.

Méthode. Un dispositif expérimental en blocs complètement randomisés avec trois répétitions par traitement a été mis en place. Les traitements analysés sont, premièrement, des cultures associées M. esculenta var. 'Bonoua Blanc'/L. siceraria avec les semis de la cucurbite à $0\left(\mathrm{CAT}_{0}\right), 15\left(\mathrm{CAT}_{15}\right), 30\left(\mathrm{CAT}_{30}\right)$ et $45\left(\mathrm{CAT}_{45}\right)$ jours après la mise en place du manioc ; deuxièmement, des semis de cultures pures de la cucurbite aux mêmes dates que pour les cultures associées $\left(\mathrm{CPT}_{0}, \mathrm{CPT}_{15}\right.$, $\mathrm{CPT}_{30}$ et $\mathrm{CPT}_{45}$ ) et troisièmement, une plantation de culture pure de manioc à 0 jour $(\mathrm{CP})$. Neuf caractères agronomiques chez la cucurbite et huit chez le manioc ont été analysés.

Résultats. Les paramètres agronomiques évalués sont les composantes de rendement du manioc qui varient avec l'augmentation du temps de décalage de semis de la cucurbite. Cependant, les performances de la cucurbite diminuent avec cette augmentation du décalage de semis, que ce soit en culture associée ou en culture pure. Malgré une productivité élevée lorsque la cucurbite est simultanément semée avec le manioc, elle reste faible comparativement à la culture pure de la cucurbite.

Conclusions. Dans une association cucurbite Lagenaria siceraria et manioc Manihot esculenta, la production n'est pas bénéfique aux cultures prises individuellement. Cependant, l'utilisation de la cucurbite comme plante de couverture en interculture permet de conserver l'humidité du sol et de gérer les surfaces cultivables et les adventices pour en faire bénéficier le manioc en association.

Mots-clés. Culture associée, système cultural, calendrier cultural, rendement.

Effect of sowing dates on the agronomic performance of the intercrops: Lagenaria siceraria (Molina) Standl. (Cucurbitaceae) and Manihot esculenta Crantz (Euphorbiaceae)

Description of the subject. The intercropping system is a beneficial method, which is widely used in tropical areas for sustainable soil fertility management, and has been found to improve both the yields of traditional agriculture and the income of small producers.

Objectives. The aim of this study was to compare and evaluate the effect of associated culture and pure culture of cucurbit Lagenaria siceraria creeping plant and the variety of cassava Manihot esculenta var. 'Bonoua Blanc' upright plant on the agronomic performance of each crop. 
Method. An experiment was established in randomized complete block design with three replicates per treatment. The treatments compared were firstly, associated crops M. esculenta var. 'Bonoua Blanc' / L. siceraria with cucurbit seedlings at 0 days $\left(\mathrm{CAT}_{0}\right), 15$ days $\left(\mathrm{CAT}_{15}\right), 30$ days $\left(\mathrm{CAT}_{30}\right)$ and 45 days $\left(\mathrm{CAT}_{45}\right)$ after cassava; secondly, pure cultures of cucurbit on the same dates as for associated crops $\left(\mathrm{CPT}_{0}, \mathrm{CPT}_{15}, \mathrm{CPT}_{30}\right.$ and $\left.\mathrm{CPT}_{45}\right)$, and thirdly, a pure culture of cassava at 0 days $(\mathrm{CP})$. Nine agronomic characteristics were analyzed in cucurbit and eight in cassava. Results. The agronomic parameters evaluated were the yield components of cassava, which varied with the increase in seedling time of cucurbit. However, the performance of cucurbit decreased with the increase in seedlings either in the associated crop or in pure culture. Performance also decreased sharply for the same sowing dates in pure culture. Despite high productivity when cucurbit was simultaneously planted with cassava, it remained low compared to the cucurbit pure crop.

Conclusions. Intercropping of cucurbit L. siceraria and cassava M. esculenta was not found to be beneficial to the yield of the individual crops. However, the use of cucurbit as an intercropping cover crop helps to conserve soil moisture and to manage arable land and weeds for the benefit of cassava crops.

Keywords. Intercropping, cropping system, cropping calendar, yield.

\section{INTRODUCTION}

Depuis les indépendances, le développement agricole des pays africains de la zone tropicale est axé sur les principales cultures d'exportation (café, cacao, coton, hévéa, palmier à huile, etc.) et les cultures vivrières (ignames, arachides, maïs, bananes, etc.). Ces cultures font l'objet de politiques agricoles bien élaborées aussi bien pour la production que pour la vente, au détriment des plantes mineures très souvent sous-exploitées et négligées. L'importance de ces plantes d'intérêt local tant sur le plan alimentaire qu'économique est reconnue par les décideurs politiques et les organismes d'aide au développement (Delang, 2006 ; Brittaine \& Lutaladio, 2010 ; Ebert, 2014 ; Nandal \& Bhardwaj, 2014). Par ailleurs, des travaux ont montré des stratégies de valorisation de ces plantes (Barnaud et al., 2013 ; Jain \& Gupta, 2013). Elles représentent un atout considérable pour l'accroissement du revenu des paysans, d'autant plus que leur valeur marchande est parfois plus élevée que celle des cultures dites d'exportation (Ebert, 2014). C'est le cas des cucurbitacées oléagineuses très prisées dans plusieurs pays d'Afrique de l'Ouest et du Centre. La richesse des graines des cucurbites oléagineuses en lipides et en protéines (Loukou et al., 2007) et les nombreuses vertus thérapeutiques qui leur sont attribuées (Ahmad et al., 2011 ; Rajput et al., 2011 ; Irshad et al., 2013) leur confèrent une place de choix dans la résolution des problèmes de santé et de qualité de l'alimentation, surtout en zone rurale. Parmi les espèces de cucurbites, Lagenaria siceraria (Molina) Standl. est la plus répandue et appréciée des populations. De par leur nature rampante, ces plantes assurent une protection efficace du sol contre l'érosion et la prolifération des mauvaises herbes par l'obscurité qu'elles créent (Zuofa et al., 1992) et par la compétition pour les ressources du milieu (Hauggaard-Nielsen et al., 2006). Malgré leur importance alimentaire et économique, le rendement des cucurbites oléagineuses demeure relativement faible, notamment du fait du manque d'itinéraires techniques de production améliorés.

Pour permettre aux producteurs d'augmenter leur production, il s'avère opportun de promouvoir et de valoriser L. siceraria. En effet, très cultivée en Côte d'Ivoire et dans la sous-région, les graines de l'espèce font l'objet d'un commerce florissant sur les marchés locaux (prix moyen de 2,30 Eur. $\cdot \mathrm{kg}^{-1}$ ) (Zoro Bi et al., 2003). Par conséquent, la culture de cette cucurbite représente une source potentielle de devises additionnelles pour les paysans. Vu l'importance de cette espèce, la mise en place d'un programme d'amélioration des systèmes de production agricole et la promotion de sa culture en milieu paysan deviennent un impératif. Ainsi, des missions exploratoires organisées dans le but de valoriser cette plante en Côte d'Ivoire ont révélé que la production de L. siceraria est répandue dans les zones Centre et Est. Ces deux régions sont caractérisées par la culture massive de plantes vivrières telles que le maïs, l'igname, le taro, les légumes, l'arachide, et de plantes annuelles ou pérennes comme le coton, le café et le cacao (Zoro Bi et al., 2003). Dans ces zones, pour lutter contre l'enherbement de ces cultures, cellesci sont fréquemment cultivées en association avec L. siceraria (Zuofa et al., 1992). Par ailleurs, dans la zone Sud du pays, la cucurbite est régulièrement associée au manioc (Manihot esculenta Crantz). Dans les associations culturales, plusieurs facteurs culturaux, dont la date de semis des plantes associées, doivent être pris en compte pour garantir un meilleur rendement. En effet, le contrôle adéquat des dates de semis est une approche qui est suggérée pour minimiser la compétition interspécifique inévitable lorsque deux espèces sont associées (Powers et al., 1994) et améliorer l'utilisation des ressources et, par conséquent, les rendements. Cette étude s'inscrit dans le cadre de l'optimisation de l'itinéraire technique pour accroitre la production de L. siceraria lorsque celle-ci est cultivée en association avec le manioc. 


\section{MATÉRIEL ET MÉTHODES}

\subsection{Site d'expérimentation}

Les expérimentations ont été conduites sur le site de l'Université Nangui Abrogoua (UNA) à Abidjan (Côte d'Ivoire). Abidjan est située entre $5^{\circ} 17^{\prime}$ et $5^{\circ} 31^{\prime}$ de latitude nord et entre $3^{\circ} 45^{\prime}$ et $4^{\circ} 22^{\prime}$ de longitude ouest. Les données de la pluviométrie, de la température et de l'humidité relative de la zone d'Abidjan accessibles gratuitement sur le site www. tutiempo.net ont permis de faire une étude de celles-ci. Cette étude a montré que notre site d'étude est caractérisé par une grande saison de pluie qui se situe entre mars et juillet avec un pic de $673 \mathrm{~mm}$ de pluie en juin 2011 (Figure 1). La petite saison de pluie quant à elle s'étend de septembre à décembre avec $280 \mathrm{~mm}$ de pluie en octobre 2012. S'agissant de la grande saison sèche, elle couvre les mois de janvier et février avec une température maximale de $27,87^{\circ} \mathrm{C}$ en février. Par ailleurs, la durée de la petite saison sèche est d'un mois (aout), avec $24,4{ }^{\circ} \mathrm{C}$. Le site des essais est une jachère caractérisée par une végétation arbustive composée de Panicum maximum Jacq. (Poacées), Eupatorium odoratum (L.) King \& H.E. Robins (Astéracées), Imperata cylindrica (L.) Beauv. (Poacées), Axonopus compressus (Sw.) P.Beauv. (Poacées), Ageratum conyzoides (L.) L. (Astéracées) et Cynodon dactylon (L.) Pers. (Poacées). Le sol est ferralitique, profond à texture sablo-argileuse et à structure grumeleuse (Yao-Kouamé \& Kane, 2008).

\subsection{Matériel végétal}

Le matériel végétal utilisé était constitué des boutures de Manihot esculenta var. 'Bonoua blanc' (BB) dont la durée du cycle est de 12 mois. Elles ont été collectées dans le village d'Ahoutoue situé dans le Sud de la Côte d'Ivoire à 38 km à l'Est d'Abidjan. Cette variété se ramifie très vite et les rameaux sont très proches de la surface du sol. Elle a des axes sylleptiques qui tirent leur origine de la transformation des méristèmes terminaux en méristèmes floraux. À chaque floraison, trois branches se développent simultanément, lui donnant un aspect trichotomique. Elle est à tubérisation tardive. Les tiges saines ont été sélectionnées sur des plantes âgées de 12 mois. À l'aide d'un sécateur régulièrement désinfecté à l'alcool, les coupes ont été faites à $90^{\circ}$ afin d'assurer une section circulaire à la base de la bouture et une répartition régulière des racines basales (Raffaillac, 1992). Les boutures ont une longueur de $25 \mathrm{~cm}$ avec au moins cinq à six nœuds où sont fixés les bourgeons axillaires.
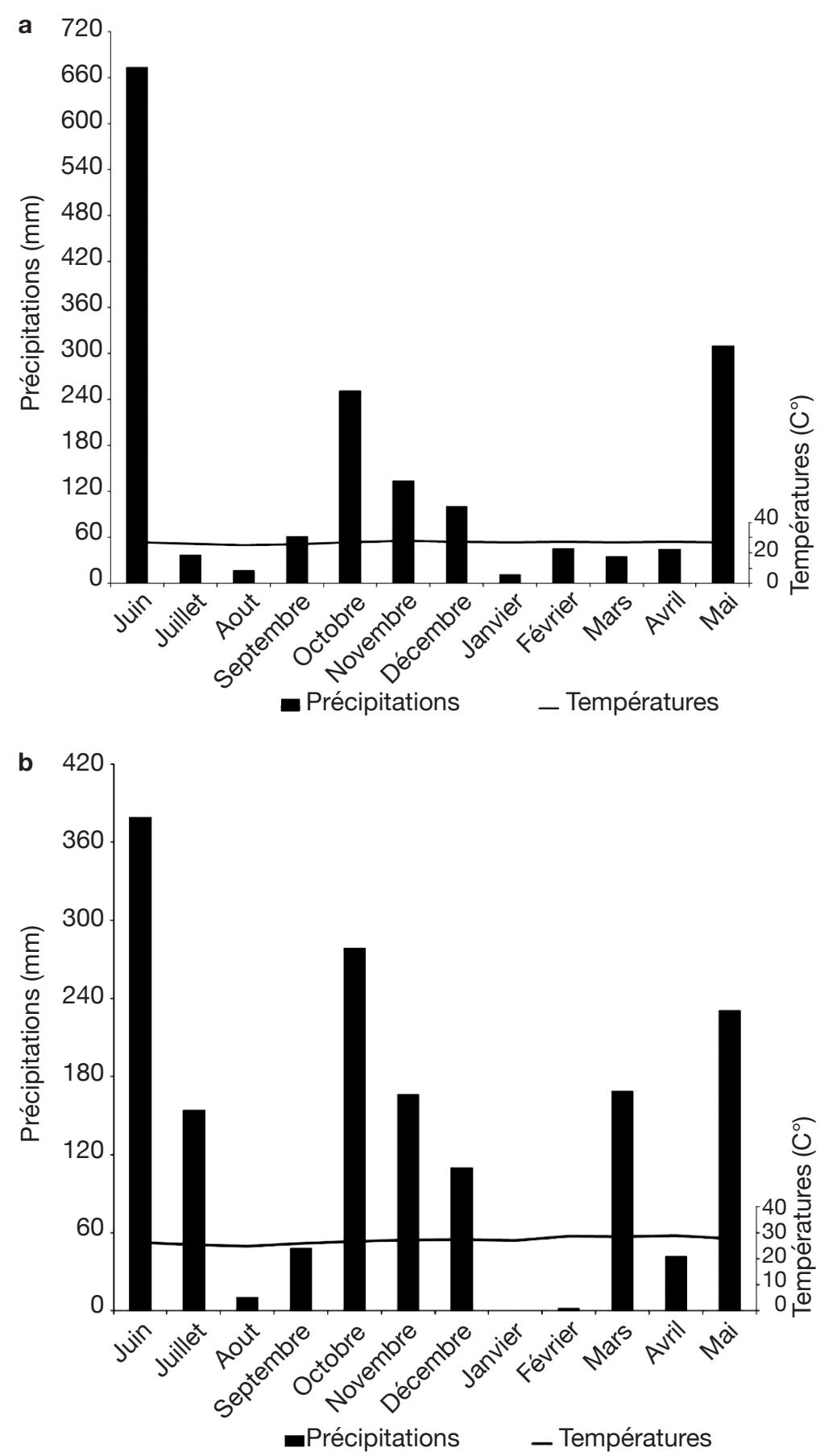

Figure 1. Diagramme ombrothermique du district d'Abidjan établi pour la période juin 2011-mai 2012 (a) et la période juin 2012-mai 2013 (b) - Ombrothermic chart of the district of Abidjan established for the period of June, 2011-May, 2012 (a) and the period of June, 2012-May, 2013 (b). 
L'espèce de cucurbite sélectionnée est $L$. siceraria, cultivar à baies rondes. La reproduction se fait par graines et le cycle de culture s'étend sur 90 à 120 jours selon le cultivar (Zoro Bi et al., 2003). Les baies contiennent des graines de petites tailles qui se caractérisent par la présence de calottes dans la partie distale. Les graines utilisées sont issues de la collection de l'Unité de Phytotechnie et Amélioration Génétique (UPAG) de l'UNA. Ce matériel végétal est une accession provenant d'une mission de collectes réalisée dans la zone agro-écologique sud de la Côte d'Ivoire où la culture de cette espèce est fréquente et régulière.

Après défrichage de la parcelle, le sol a été ameubli à la houe par un labour profond de 20 à $30 \mathrm{~cm}$ environ un mois avant de planter les boutures de manioc à une profondeur de 15 à $20 \mathrm{~cm}$. Les graines de L. siceraria ont été enterrées à 2 ou $3 \mathrm{~cm}$ de profondeur. Toutes les cultures ont été faites sans fertilisation. Trois traitements insecticides au Cypercal 50 EC (cyperméthrine) ont été effectués sur les cucurbites pour réduire l'impact des insectes. Le premier a lieu dès que $50 \%$ des plantes de cucurbite ont commencé à ramper. Le second a été appliqué, au début de la floraison mâle et enfin, le troisième au moment de la formation des premiers fruits. Un désherbage régulier a été effectué pour éviter toute compétition entre les adventices et les plantes.

\subsection{Dispositif expérimental}

Un dispositif expérimental en blocs complètement randomisés avec trois répétitions par traitement a été mis en place. Quatre traitements de culture associée ont été appliqués sur une parcelle de $1530 \mathrm{~m}^{2}(30 \mathrm{~m} \mathrm{X}$ $51 \mathrm{~m})$. Chaque traitement correspondait à une sousparcelle de $5 \mathrm{~m} \times 5 \mathrm{~m}\left(25 \mathrm{~m}^{2}\right)$. Les sous-parcelles consécutives étaient séparées par une bande de terrain nu large de $2 \mathrm{~m}$ et régulièrement sarclées. Les traitements étaient des cultures associées M.esculenta var. 'Bonoua Blanc'/L. siceraria avec quatre temps de semis de la cucurbite : $0\left(\mathrm{CAT}_{0}\right), 15\left(\mathrm{CAT}_{15}\right), 30$ $\left(\mathrm{CAT}_{30}\right)$ et $45\left(\mathrm{CAT}_{45}\right)$ jours après la mise en place du manioc. Chaque sous-parcelle a reçu 25 boutures plantées sur 5 lignes de 5 poquets avec $1 \mathrm{~m}$ entre les points de plantation et les lignes. Lagenaria siceraria a été semée entre les lignes de manioc, à raison d'un point de semis par $\mathrm{m}^{2}$, soit un total de 16 points sur les sous-parcelles en association.

De plus, des cultures pures ont été réalisées aux mêmes dates qu'en culture associée à $0,15,30$ et 45 jours pour les semis de L. siceraria $\left(\mathrm{CPT}_{0}, \mathrm{CPT}_{15}\right.$, $\mathrm{CPT}_{30}$ et $\mathrm{CPT}_{45}$ ) et seulement au temps $\mathrm{T}_{0}$ pour la plantation du manioc (CP). Chaque sous-parcelle abritant les cultures pures a comporté 5 lignes de semis distantes de $1 \mathrm{~m}$ les unes des autres. Les points de semis sur les lignes sont également distants de $1 \mathrm{~m}$. $\mathrm{Au}$ total, chaque parcelle élémentaire contenait soit 25 pieds de cucurbite, soit 25 pieds de manioc.

L'expérimentation a été répétée deux fois sur le même site. La première répétition a été effectuée de juin 2011 à mars 2012 et la seconde, de juin 2012 à mars 2013. La pluviométrie totale des deux périodes de culture était de $1368 \mathrm{~mm}$ et $1365 \mathrm{~mm}$ respectivement.

\subsection{Paramètres agronomiques mesurés}

Pour le manioc, huit caractères agronomiques (Fukuda et al., 2010) ont été examinés dans cette étude. Les observations ont été effectuées sur toutes les plantes dans chaque parcelle élémentaire. La récolte de ces plantes a eu lieu 10 mois après la plantation selon l'approche de Lenis et al. (2006). Après la détermination du nombre de tiges issues d'une même bouture (NTPl), le nombre de feuilles par plante $(N F P l)$ a été déterminé en multipliant le nombre d'apex par plante par le nombre moyen de feuilles par apex déterminé selon la méthodologie adoptée par Daellenbach et al. (2005). Enfin, le diamètre de la base de la tige (DiTi) et la longueur totale de la tige (LoTi) ont été mesurés sur la tige la plus développée de la plante. La méthodologie des mesures suit celle décrite par Ambang et al. (2007). Ces paramètres représentent les paramètres de croissance. À la récolte, les plantes ont été déracinées et le nombre de racines tubérisées (NRTP), le poids moyen d'une racine tubérisée $(P I R T P)$ et le poids des racines tubérisées par plante $(P R T P)$ ont été notés. La méthodologie des mesures suit celle décrite par Aniekwe et al. (2004). Ces paramètres représentent les paramètres de rendement. En outre, le rendement frais à l'hectare (RdHa) a été calculé. Il a été estimé comme le produit du poids moyen des racines tubérisées par plante et le nombre des plantes calculé pour 1 ha, soit 10000.

Chez la cucurbite L. siceraria, neuf paramètres agronomiques sélectionnés parmi les descripteurs des cucurbites (Staub \& Frederick, 1988 ; Koffi et al., 2009) ont été mesurés : la longueur de la plante $(L o P l)$, le nombre de fruits par plante $(N F r P)$, le poids des fruits $(P o F r)$, le diamètre des fruits $(D i F r)$, la cavité de la loge des graines (CLGr) des baies, la hauteur des fruits $(\mathrm{HaFr})$, le nombre de graines par fruit $(\mathrm{NGFr})$, le poids de 100 graines $(P 100)$ par fruit et le rendement en graines $(\mathrm{RdHa})$ selon la formule suivante :

$$
R d H a=\frac{N G F r \times P 100}{100} \times N F r P \times 10000
$$




\subsection{Indice de compétition}

Compte tenu des décalages de semis opérés dans cette expérimentation, un seul cycle de la cucurbite a été possible pendant le cycle du manioc. L'espace disponible après la récolte de la cucurbite n'ayant pas été exploité, le taux de surface équivalente (LER) a été utilisé pour évaluer les avantages de l'association cucurbite-manioc (Willey \& Osiru, 1972). Le LER compare la production des cultures associées et celle de leur équivalent en culture pure, à surface totale égale et indique l'efficience de l'utilisation des ressources environnementales en association culturale comparée au système monocultural (Mead \& Willey, 1980). Il correspond à la surface nécessaire pour obtenir, à partir des deux cultures pures, la même production qu'en association. Une valeur de LER $>1$ indique un avantage du mélange d'espèces par rapport à une culture de chaque espèce séparément, avec une meilleure utilisation des ressources dans l'association. Au contraire, si LER < 1, l'association culturale n'est pas avantageuse (Dhima et al., 2007) et la compétition interspécifique est plus forte que les interactions interspécifiques entre les systèmes d'association culturale (Zhang et al., 2011). Le LER a été calculé en utilisant la formule suivante :

$$
\begin{aligned}
& L E R=\left(L E R_{\text {manioc }}+L E R_{\text {cucurbite }}\right) \\
& L E R_{\text {manioc }}=\frac{R d H a_{m a}}{R d H a_{m p}} \\
& L E R_{\text {cucurbite }}=\frac{R d H a_{c a}}{R d H a_{c p}}
\end{aligned}
$$

où $R d H a_{m p}$ est le rendement frais du manioc en cultures pures, $R d H a_{c p}$ est le rendement frais de la cucurbite en cultures pures, $R d H a_{m a}$ est le rendement frais du manioc en cultures associées et $R d H a_{c a}$ est le rendement frais de la cucurbite en cultures associées.

\subsection{Analyse des données}

Les systèmes culturaux et l'agenda de semis ainsi que leur interaction ont fait chacun l'objet d'une analyse multiple de la variance (MANOVA) afin d'évaluer leur influence globale sur les paramètres mesurés. Cela a permis de sélectionner les facteurs ou interactions qui ont montré un effet significatif. Les MANOVAs ont été complétées par des tests ANOVA (Analyse de la variance à un ou deux critères de classification) afin de déterminer l'effet d'un facteur ou l'association de deux facteurs sur les paramètres agronomiques et le LER. Lorsqu'une différence significative est observée, des comparaisons multiples sont faites en effectuant le test de la plus petite différence significative (ppds). Ce test a permis d'identifier le ou les traitements qui diffèrent significativement les uns des autres (Dagnelie, 1998). La signification de la différence des moyennes est déterminée en comparant la probabilité $p$ associée à la statistique du test de Fischer-Snedecor au seuil théorique de $\alpha=0,05$. Les analyses ont été réalisées avec le logiciel SAS (SAS, 2004).

\section{RÉSULTATS}

\subsection{Effet global des systèmes culturaux et de l'agenda de semis sur la croissance et le rendement de la cucurbite et du manioc}

Le tableau 1 présente les résultats de l'analyse multivariée de la variance portant sur l'effet global des systèmes culturaux et de l'agenda de semis de la cucurbite, sur les performances agronomiques de la cucurbite et du manioc. Ce test a montré que les types de culture, l'agenda de semis et l'interaction « cultures $\times$ agenda de semis » ont un effet significatif sur la production $(p<0,05)$. Toutefois, les interactions étant significatives, les interprétations seront faites avec les tableaux des interactions (ANOVA II). Considérant les doubles interactions, un tableau pour la cucurbite et un autre pour le manioc ont été présentés.

\subsection{Effet de l'agenda de semis sur la performance agronomique de $L$. siceraria}

Le tableau 2 présente le résultat de l'ANOVA réalisée pour tester l'effet de l'interaction des systèmes de culture et de l'agenda de semis sur les paramètres agronomiques de la cucurbite. Tous les paramètres

Tableau 1. Analyse multivariée (AMOVA) réalisée pour tester l'effet global de l'agenda de semis de la cucurbite et des systèmes culturaux sur la productivité de Lagenaria siceraria et du manioc - Multivariate analysis of variance (AMOVA) performed to test the overall effect of time of planting of cucurbit and cultural systems on the productivity of Lagnenaria siceraria and cassava.

\begin{tabular}{llllll}
\hline \multirow{2}{*}{$\begin{array}{l}\text { Sources de } \\
\text { variation }\end{array}$} & \multicolumn{2}{l}{ Lagenaria siceraria } & & \multicolumn{2}{l}{ Manioc } \\
\cline { 2 - 3 } \cline { 5 - 6 } d.I. & F & & d.l. & F \\
\hline Cultures & 11 & $38,21 * * *$ & & 10 & $17,88 * * *$ \\
Agenda de semis & 33 & $44,24 * * *$ & & 30 & $4,53 * * *$ \\
Cultures $\times$ agenda & 33 & $12,02 * * *$ & 30 & $4,53 * * *$ \\
\hline
\end{tabular}

d.1. : degré de liberté - degree of freedom ; *** : différence significative $(p<0,001)-$ significative difference $(\mathrm{p}<0.001)$. 


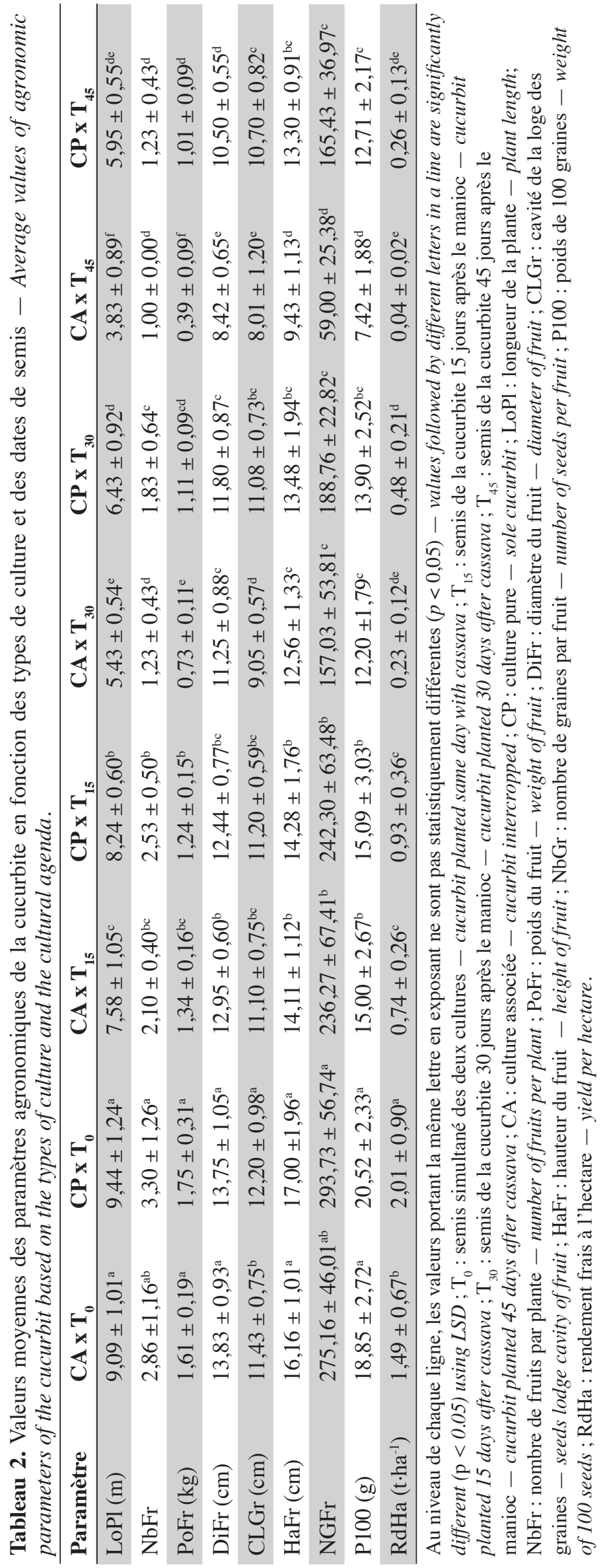

sont sous l'influence de l'interaction système de culture et agenda de semis $(p<0,001)$. Les valeurs des paramètres de croissance et de rendement ont été plus élevées lorsque $L$. siceraria est semée en même temps que le manioc, en culture pure comme en culture associée, avec toutefois des valeurs plus élevées pour la culture associée $\left(\mathrm{CAT}_{0}\right)$. Par contre, les plus faibles valeurs ont été observées lorsque L. siceraria est associée au manioc avec un décalage de 45 jours. Cependant, lorsque la cucurbite est semée 15 jours après le manioc, le rendement varie de $1,49 \mathrm{t} \cdot \mathrm{ha}^{-1}$ (traitement $\mathrm{CAT}_{0}$ ) à $0,74 \mathrm{t} \cdot \mathrm{ha}^{-1}$ (traitement $\mathrm{CAT}_{15}$ ), soit une perte de $0,75 \mathrm{t} \cdot \mathrm{ha}^{-1}(50,33 \%$ de perte). Par ailleurs, la baisse de rendement est plus accentuée avec un temps de décalage de semis de la cucurbite plus élevé par rapport au temps de semis du manioc. En effet, les rendements en graines calculés pour les traitements $\mathrm{CAT}_{30}\left(0,23 \mathrm{t} \cdot \mathrm{ha}^{-1}\right)$ et $\mathrm{CAT}_{45}(0,04 \mathrm{t}$. ha $^{-1}$ ) mettent en évidence des pertes de $84,56 \%$ et $97,31 \%$ respectivement. D'une manière générale, les valeurs des paramètres décroissent avec l'augmentation du temps qui sépare la plantation du manioc et le semis de L. siceraria, que ce soit en culture associée ou en culture pure.

\subsection{Effet de l'agenda de semis sur la performance agronomique du manioc}

Les résultats de l'ANOVA réalisée pour tester l'effet de l'interaction de l'agenda de semis et des systèmes de culture sur les paramètres de croissance et de rendement de la variété non améliorée de manioc 'BB' sont repris au tableau 3. Pour le nombre de tiges par plante $(N T P l)$, il n'y a pas d'effet significatif entre les différents traitements $(p=0,229)$. Par contre, l'agenda de semis de la cucurbite a influencé les sept autres paramètres analysés $(p<0,05)$. Les différences observées sont dues au fait que les variables $\mathrm{NFPl}, \mathrm{DiTi}$, LoTi, NRTP, PIRTP, PRTP et RdHa présentent les valeurs les plus élevées lorsque la cucurbite est semée au moins 15 jours après la plantation du manioc ou lorsque le manioc est en culture pure. Les plantes produisent des tiges bien vigoureuses portant de nombreuses feuilles, beaucoup de racines tubérisées par plante avec des valeurs de rendement élevées. La tendance générale de ces résultats révèle la faible croissance et la faible productivité du manioc lorsque les deux cultures sont installées simultanément.

\subsection{Indice de compétition}

L'analyse des résultats consignés dans la figure 2 révèle que les décalages de semis influencent 

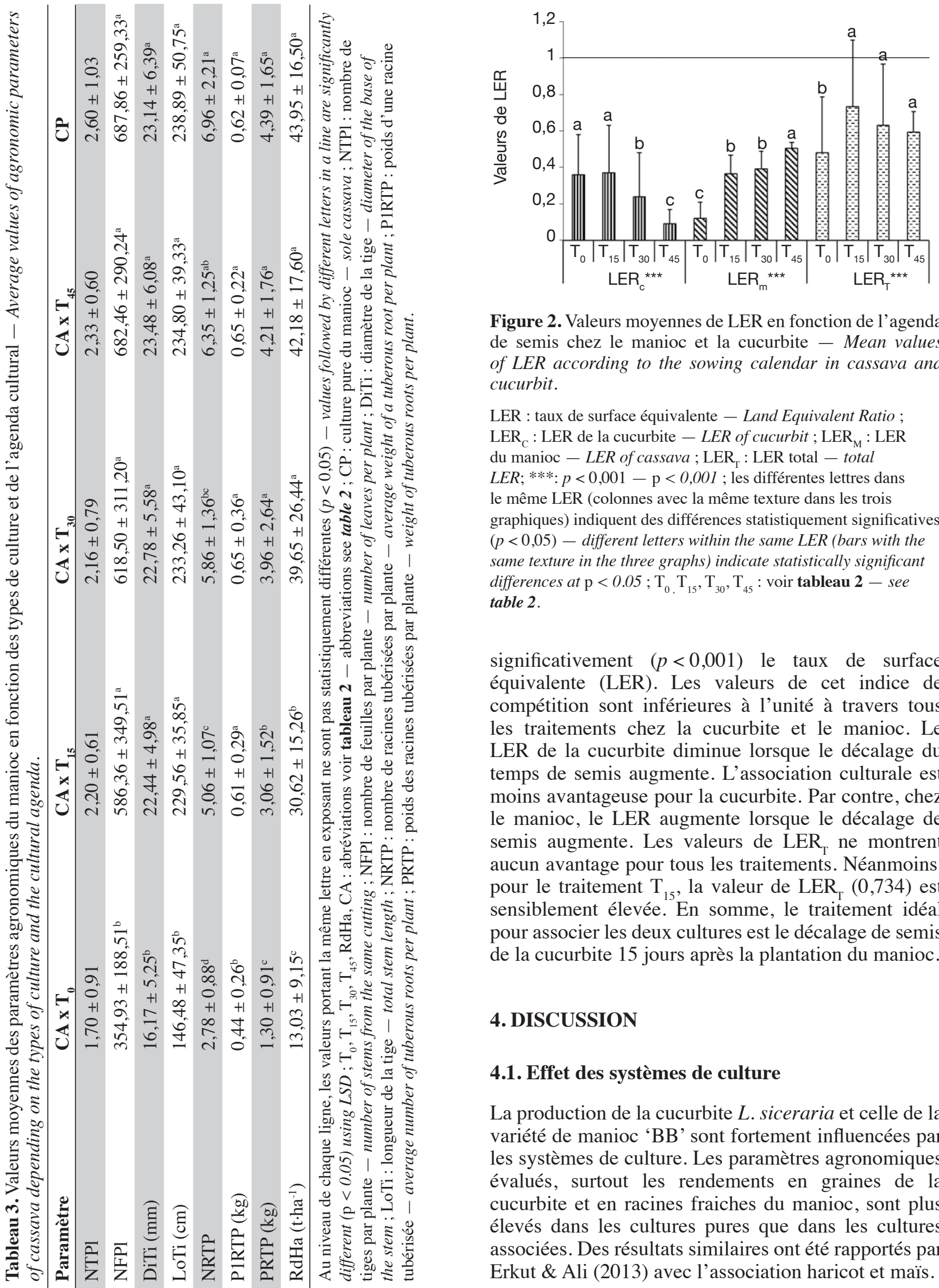

Figure 2. Valeurs moyennes de LER en fonction de l'agenda de semis chez le manioc et la cucurbite - Mean values of LER according to the sowing calendar in cassava and cucurbit.

LER : taux de surface équivalente - Land Equivalent Ratio ; LER $_{\mathrm{C}}$ : LER de la cucurbite - LER of cucurbit ; LER $_{\mathrm{M}}$ : LER du manioc - LER of cassava LER $_{\mathrm{T}}$ : LER total - total $L E R$; ***: $p<0,001-\mathrm{p}<0,001$; les différentes lettres dans le même LER (colonnes avec la même texture dans les trois graphiques) indiquent des différences statistiquement significatives $(p<0,05)$ - different letters within the same LER (bars with the same texture in the three graphs) indicate statistically significant differences at $\mathrm{p}<0.05 ; \mathrm{T}_{0}, \mathrm{~T}_{15}, \mathrm{~T}_{30}, \mathrm{~T}_{45}$ : voir tableau $2-$ see table 2.

significativement $(p<0,001)$ le taux de surface équivalente (LER). Les valeurs de cet indice de compétition sont inférieures à l'unité à travers tous les traitements chez la cucurbite et le manioc. Le LER de la cucurbite diminue lorsque le décalage du temps de semis augmente. L'association culturale est moins avantageuse pour la cucurbite. Par contre, chez le manioc, le LER augmente lorsque le décalage de semis augmente. Les valeurs de LER $_{\mathrm{T}}$ ne montrent aucun avantage pour tous les traitements. Néanmoins, pour le traitement $\mathrm{T}_{15}$, la valeur de $\operatorname{LER}_{\mathrm{T}}(0,734)$ est sensiblement élevée. En somme, le traitement idéal pour associer les deux cultures est le décalage de semis de la cucurbite 15 jours après la plantation du manioc.

\section{DISCUSSION}

\subsection{Effet des systèmes de culture}

La production de la cucurbite $L$. siceraria et celle de la variété de manioc 'BB' sont fortement influencées par les systèmes de culture. Les paramètres agronomiques évalués, surtout les rendements en graines de la cucurbite et en racines fraiches du manioc, sont plus élevés dans les cultures pures que dans les cultures associées. Des résultats similaires ont été rapportés par Erkut \& Ali (2013) avec l'association haricot et maïs. 
La diminution des valeurs des paramètres agronomiques de la cucurbite et du manioc testés en cultures associées s'expliquerait par la compétition pour les ressources environnementales disponibles (Powers et al., 1994). Ainsi, les plantes disposeraient de peu de ressources en cultures associées par rapport aux cultures pures. Aussi, Mutsaers et al. (1993) ont souligné que les avantages de l'association tendent à diminuer parce que le temps de croissance de la cucurbite dépasse les 90 jours. Ces auteurs ont en outre rapporté que le manioc est la composante dominante lorsqu'il est associé à des espèces rampantes comme les cucurbites. En effet, l'indice de surface foliaire (LAI) du manioc influence grandement le rendement de la cucurbite associée. Des essais d'association manioc/maïs/okra (Abelmoschus esculentus [L.] Moench/Citrullus lanatus [Thunb.] Matsum. \& Nakai [cucurbite]) menés par Ikeorgu (1991) ont montré une diminution du rendement en graines de $78 \%$ de la cucurbite sous un LAI élevé du manioc, tandis que leurs rendements en graines augmentent significativement sous un LAI plus faible du manioc.

Les résultats montrent que le $\mathrm{LER}_{\mathrm{C}}$ décroît de 0,36 à 0,09 alors que le $\operatorname{LER}_{\mathrm{M}}$ croît de 0,12 à 0,50 de $\mathrm{T}_{0}$ à $\mathrm{T}_{45}$. Dans cette pratique culturale, le LER était significativement inférieur à 1,00 indiquant un désavantage de l'association par rapport aux cultures pures, en termes d'utilisation des ressources environnementales pour la croissance des plantes (Mead \& Willey, 1980). Dans un autre cas, les faibles valeurs de LER observées révélaient que la compétition était plus grande que l'interaction interspécifique ou complémentarité, si bien que l'association aboutissait à une utilisation moins efficiente de l'espace par les espèces. Ainsi, la faible performance en termes de LER est obtenue dans les communautés de plantes à grande compétition (Nassab et al., 2011).

Des cas de valeurs de LER plus grandes que 1,00 ont été signalés dans l'analyse des rendements du sorgho (Sorghum bicolor) et de la gourde (Lagenaria siceraria) associés (Chimonyo et al., 2016), du manioc et des légumes associés (Islami et al., 2011 ; Mbah \& Ogidi, 2012 ; Hidoto \& Loha, 2013), et du maïs (Zea mays) associé à la cucurbite (Citrullus lanatus) et au manioc (Ijoyah et al., 2012).

\subsection{Effet de l'agenda de semis sur la productivité de la cucurbite et du manioc}

Le semis simultané cucurbite-manioc a montré les meilleures performances de croissance et de développement chez la cucurbite, tandis que chez le manioc, les plus faibles valeurs ont été observées. Une tendance similaire avait été rapportée par Adipala et al. (2002) dans une étude montrant que le décalage de semis du niébé (Vigna unguiculata [L.]
Walp.) par rapport au maïs (Zea mays L.) ralentit la croissance et le rendement du niébé. Kalonda (1993) a également conduit une expérience d'association culturale niébé-cotonnier et avait rapporté que l'introduction précoce du niébé après le semis du cotonnier accroît son rendement, alors qu'elle réduit la production du coton-graine. Des résultats similaires ont été obtenus par Hirpa (2013) qui avait montré que l'effet du temps des cultures intercalaires de légumineuses sur le rendement du maïs avait un effet significatif sur le nombre d'épis de maïs par $\mathrm{m}^{2}$. Ainsi, dans cette étude, la mauvaise performance du manioc en culture associée avec la cucurbite serait due à un développement initial lent du manioc (Amanullah et al., 2007) et à une plus grande capacité concurrentielle de la cucurbite. Cette dernière est récoltée avant que le manioc ne développe la totalité de sa canopée (Olasantan et al., 1996). En effet, les deux premiers mois du développement du manioc correspondent à la phase d'installation. Pendant cette phase, la croissance végétative est ralentie au profit de celle des racines (Silvestre \& Arraudeau, 1983). Ainsi semée tôt, la cucurbite germe et croît rapidement. Cette plante vigoureuse et rampante, en s'étalant, a non seulement réduit les adventices en les étouffant (Djè $\mathrm{Bi}$ et al., 2011) mais a également entravé le développement du manioc. La période de compétition interspécifique étant inévitable lorsque des espèces sont associées (Ghaffarzadeh et al., 1997), l'intégration précoce de la cucurbite au manioc aurait retardé cette période et favorisé son développement. Elle aurait disposé d'éléments nutritifs au moment approprié, ce qui en a amélioré ses performances (Powers et al., 1994).

Toutefois, dans l'association manioc-cucurbite, les performances du manioc s'améliorent avec le retard d'introduction de la cucurbite. Par contre, les performances agronomiques de L. siceraria diminuent avec le retard de son introduction dans le système de production du manioc. Les résultats observés dans cette étude sont similaires à ceux de Ifenkwe \& Odurukwe (1990) qui avaient introduit le maïs à $0,1,2,3$ et 4 semaines après plantation de la pomme de terre. Ces chercheurs avaient remarqué un accroissement des rendements de la pomme de terre avec le retard d'introduction du maïs. Cependant, ce retard diminuait les rendements du maïs. Selon également Hirpa (2013), le maïs semé 8 semaines avant les légumineuses avait produit le plus grand nombre d'épis par $\mathrm{m}^{2}$.

Dans cette étude, le faible rendement de la cucurbite obtenu avec le plus long décalage (30 et 45 jours) est probablement dû à l'effet de l'ombrage que le manioc crée à cette période de son cycle de développement. En effet, la période comprise entre le deuxième et le quatrième mois après plantation correspond à la phase de développement du système aérien du manioc. 
C'est aussi à cette période que le système racinaire devient fonctionnel et que la croissance caulinaire est intensément reprise (Silvestre \& Arraudeau, 1983). Ainsi, l'ombrage créé par le manioc aurait inhibé la croissance de la cucurbite. La faible croissance de la cucurbite peut être causée soit par la réduction de la qualité et de la quantité de lumière due à la canopée, soit à la température du sol (Hauggaard-Nielsen et al., 2006). D'ailleurs, les pieds de L. siceraria observés dans ces conditions étaient particulièrement étiolés. L'étiolement de la cucurbite réduit sa capacité photosynthétique (Moser et al., 2005), c'est-à-dire la production de molécules organiques et leur accumulation au cours du remplissage des graines. Cela a pour conséquence la production de petites baies contenant peu de graines et qui sont légères. En outre, les semis décalés de 30 à 45 jours, même en culture pure, s'étant effectués au mois de juillet, le début du cycle de reproduction des plantes s'est situé au mois d'aout qui correspond à la petite saison sèche en Côte d'Ivoire. Les pluies de la période de culture ont été moins abondantes et moins fréquentes. Cependant, Wang et al. (2004) ont montré que des suppléments d'eau de 23, 45 et $68 \mathrm{~mm}$ accroissent le nombre et le poids des fruits de la cucurbite de 21, 26 et $38 \%$ respectivement, comparés aux parcelles non irriguées. Ainsi, l'insuffisance d'eau peut défavoriser la croissance et le développement des fruits, ce qui peut conduire aux faibles rendements.

\section{CONCLUSIONS}

Dans les systèmes de polyculture, les rendements améliorés des cultures associées sont de bons indicateurs pour orienter les stratégies d'association des plantes en milieu rural (Achigan-Dako et al., 2006). Ainsi, la production de la cucurbite L. siceraria et du manioc peut être optimisée par la pratique de leur association avec l'adoption d'un décalage de semis adéquat entre les deux cultures. Pour obtenir les meilleures performances de L. siceraria lors de son association au manioc, il faudra la semer 15 jours après le manioc. L'augmentation de ce délai a entrainé la baisse de la productivité de la cucurbite. Par contre, elle a favorisé la réduction de la compétition exercée sur le manioc. L'association de la cucurbite 15 jours après la plantation du manioc n'a pas été intéressante au regard du rendement des cultures $\left(\mathrm{LER}_{\mathrm{T}}<1\right)$. Cependant, son utilisation comme plante de couverture en interculture a permis de conserver l'humidité du sol et de gérer les surfaces cultivables et les adventices (Gunton et al., 2011) pour en faire bénéficier le manioc en association. La vulgarisation d'un tel système devrait être envisagée auprès des utilisateurs finaux que sont les producteurs ruraux. Cela requiert, au préalable, des essais multilocaux. Il s'agira de rapporter les résultats d'une étude comparative de production du manioc et de L. siceraria, suivant les pratiques paysannes et un itinéraire technique dans lequel sera pris en compte le délai de 15 jours correspondant au meilleur temps d'intégration de la cucurbite dans la culture du manioc.

\section{Remerciements}

Cette étude a été réalisée grâce au financement de l'Académie de Recherche et d'Enseignement Supérieur (ARES, Bruxelles, Belgique).

\section{Bibliographie}

Achigan-Dako G.E. et al., 2006. Évaluation agronomique de trois espèces de Egusi (Cucurbitaceae) utilisées dans l'alimentation au Bénin et élaboration d'un modèle de prédiction du rendement. Biotechnol. Agron. Soc. Environ., 10(2), 121-129.

Adipala E., Ocaya C.P. \& Osiru D.S.O., 2002. Effect of time of planting cowpea (Vigna unguiculata (L.) Walp.) relative to maize (Zea mays L.) on growth and yield of cowpea. Tropicultura, 20(2), 49-57.

Ahmad I., Irshad M. \& Rizvi M.M.A., 2011. Nutritional and medicinal potential of Lagenaria siceraria. Int. J. Veg. Sci., 17, 157-170.

Amanullah M.M., Somasundaram E., Vaiyapuri K. \& Sathyamoorthi K., 2007. Intercropping in cassava. A review. Agric. Rev., 28(3), 179-187.

Ambang Z. et al., 2007. Tolérance de quelques cultivars de manioc (Manihot esculenta Crantz) et de l'espèce sauvage (Manihot glaziovii) à la mosaïque virale africaine et à la cercosporiose du manioc. Tropicultura, 25(3), 140-145.

Aniekwe N.L., Okereke O.U. \& Anikwe M.A.N., 2004. Modulating effect of black plastic mulch on the environment, growth and yield of cassava in a derived savanna belt of Nigeria. Tropicultura, 22(4), 185190.

Barnaud A. et al., 2013. From advanced to underutilized crops: making fonio benefit from research on major cereals in Africa. Acta Hortic., 979, 421-430.

Brittaine R. \& Lutaladio N., 2010. Jatropha: a small-holder bioenergy crop. The potential for pro-poor development. Roma: IFAD/FAO.

Chimonyo V.G.P., Modi A.T. \& Mabhaudhi T., 2016. Water use and productivity of a sorghum-cowpea-bottle gourd intercrop system. Agric. Water Manage., 165, 82-96.

Daellenbach G.C. et al., 2005. Plant productivity in cassavabased mixed cropping systems in Colombian hillside farms. Agric. Ecosyst. Environ., 105, 595-614.

Dagnelie P., 1998. Statistique théorique et appliquée. Vol. 2. Bruxelles : De Boeck \& Larcier. 
Delang C.O., 2006. Not just minor forest products: the economic rationale for the consumption of wild food plants by subsistence farmers. Ecol. Econ., 59, 6473.

Dhima K.V., Lithourgidis A.S., Vasilakoglou I.B. \& Dordas C.A., 2007. Competition indices of common vetch and cereal intercrops in two seeding ratio. Field Crops Res., 100, 249-256.

Djè Bi I.R., Koffi K.K., Baudoin J.-P. \& Zoro Bi I.A., 2011. Effet de la saison de culture et de la densité des plants sur les adventices et la production de la cucurbite oléagineuse Citrullus lanatus (Thunberg) Matsum. \& Nakai (Cucurbitaceae). Sci. Nat., 8(1), 85-93.

Ebert A.W., 2014. Potential of underutilized traditional vegetables and legume crops to contribute to food and nutritional security, income and more sustainable production systems. Sustainability, 6, 319-335.

Erkut P. \& Ali G., 2013. Intercropping efficiency and yields of intercropped maize (Zea mays L.) and dwarf bean (Phaseolus vulgaris L.) affected by planting arrangements, planting rates and relative time of sowing. Int. J. Curr. Microbiol. Appl. Sci., 2(11), 290-299.

Fukuda W.M.G.,Guevara C.L.,Kawuki R.\&Ferguson M.E., 2010. Selected morphological and agronomic descriptors for the characterization of cassava. Ibadan, Nigeria: International Institute of Tropical Agriculture (IITA).

Ghaffarzadeh M., Préchac F.G. \& Curse R.M., 1997. Tillage effect on soil water content and corn yield in strip intercropping system. Agron. J., 89(6), 893-899.

Gunton R.M., Petit S. \& Gaba S., 2011. Functional traits relating arable weed communities to crop characteristics. J. Veg. Sci., 22, 541-550.

Hauggaard-Nielsen H., Andersen M.K., Jørnsgaard B. \& Jensen E.S., 2006. Density and relative frequency effects on competitive interactions and resource use in peabarley intercrops. Field Crops Res., 95, 256-267.

Hidoto L. \& Loha G., 2013. Identification of suitable legumes in cassava (Manihot esculenta Crantz)-legumes intercropping. Afr. J. Agric. Res., 8, 2559-2562.

Hirpa T., 2013. Maize productivity as affected by intercropping date of companion legume crops. J. Agric. Sci., 1(5), 70-82.

Ifenkwe O.P. \& Odurukwe S.O., 1990. Potato/maize intercropping in the Jos Plateau of Nigeria. Field Crops Res., 25, 73-82.

Ijoyah M.O., Bwala R.I. \& Iheadindueme C.A., 2012. Response of cassava, maize and egusi melon in a three crop intercropping system at Makurdi, Nigeria. Int. J. Dev. Sustainability, 1, 135-144.

Ikeorgu J.E., 1991. Effects of maize and cassava on the performance of intercropped egusi melon (Citrullus lanatus (L.) Thum.) and okra (Abelmoschus esculentus (L.) Moench.) in Nigeria. Sci. Hortic., 48, 261-268.

Irshad M. et al., 2013. Antioxidant capacity and phenolic content of the aqueous extract of commonly consumed cucurbits. Int. J. Food Prop., 17, 179-186.
Islami T., Guritno B. \& Utomo W.H., 2011. Performance of cassava (Manihot esculenta Crantz) based cropping systems and associated soil quality changes in the degraded tropical uplands of East Java, Indonesia. J. Trop. Agric., 49, 31-39.

Jain S.M. \& Gupta D., 2013. Biotechnology of neglected and underutilized crops. Berlin, Germany: Springer.

Kalonda O., 1993. Cultures associées de coton (Gossypium hirsutum) et de niébé (Vigna unguiculata) au Zaïre. Cah. Agric., 2, 146-149.

Koffi K.K. et al., 2009. Morphological and allozyme variation in a collection of Lagenaria siceraria (Molina) Standl. from Côte d'Ivoire. Biotechnol. Agron. Soc. Environ., 13(2), 257-270.

Lenis J.I.et al., 2006. Leaf retention and cassava productivity. Field Crops Res., 95(2-3), 126-134.

Loukou A.L. et al., 2007. Macronutrient composition of three cucurbit species cultivated for seed consumption in Côte d'Ivoire. Afr. J. Biotechnol., 6(5), 529-533.

Mbah E.U. \& Ogidi E., 2012. Effect of soybean plant populations on yield and productivity of cassava and soybean grown in a cassava-based intercropping system. Trop. Subtrop. Agroecosyst., 15, 241-248.

Mead R. \& Willey R.W., 1980. The concept of a land equivalent ratio and advantages in yields for intercropping. Exp Agric., 16, 217-228.

Moser S.B., Feil B., Jampatong S. \& Stamp P., 2005. Effects of pre-anthesis drought, nitrogen fertilizer rate, and variety on grain yield, yield components, and harvest index of tropical maize. Agric. Water Manage., 81, 4158 .

Mutsaers H.J.W., Ezumah H.C. \& Osiru D.S.O., 1993. Cassava-based intercropping: a review. Field Crops Res., 34(3-4), 431-457.

Nandal U. \& Bhardwaj R.L., 2014. The role of underutilized fruits in nutritional and economic security of tribals: a review. Crit. Rev. Food Sci. Nutr., 54, 880-890.

Nassab M.D.A., Amonb T. \& Kaul H.-P., 2011. Competition and yield in intercrops of maize and sunflower for biogas. Ind. Crops Prod., 34, 1203-1211.

Olasantan F.O., Ezumah H.C. \& Lucas E.O., 1996. Effects of intercropping with maize on the micro-environment, growth and yield of cassava. Agric. Ecosyst. Environ., 57(2-3), 149-158.

Powers L.E., McSorley R., Dunn R.A. \& Montes A., 1994. The agroecology of a cucurbit-based intercropping system in the Yeguare Valley of Honduras. Agric. Ecosyst. Environ., 48, 139-147.

Raffaillac J.P., 1992. Enracinement de la bouture de manioc (Manihot esculenta Crantz) au cours des premières semaines de croissance. Agron. Trop., 46(4), 273-281.

Rajput M.S. et al., 2011. Fibrinolytic activity of kaempferol isolated from the fruits of Lagenaria siceraria (Molina) Standley. Nat. Prod. Res., 25, 1870-1875.

SAS, 2004. SAS for Windows. Cary, NC, USA: SAS Institute Inc. 
Silvestre P. \& Arraudeau M., 1983. Le manioc. Paris : Maisonneuse \& Larose.

Staub J.E. \& Frederick L.R., 1988. Evaluation of fruit quality in Cucumis sativus var. hardwickii (R.) Alef.derived lines. Cucurbit Genet. Cooperative Rep., 11, 25-28.

Wang Y.J., Xie Z.K., Li F. \& Zhang Z., 2004. The effect of supplemental irrigation on watermelon (Citrullus lanatus) production in gravel and sand mulched fields in the Loess Plateau of northwest China. Agric. Water Manage., 69, 29-41.

Willey R.W. \& Osiru D.S.O., 1972. Studies on mixtures of maize and beans (Phaseolus vulgaris) with particular reference to plant population. J. Agric. Sci., 79, 517529.

Yao-Kouamé A. \& Kane F., 2008. Biochemical characteristics of Lippia multiflora (Verbenaceae) leaves with respect to fertilizer applied to the soil. J. Plant Sci., 3(4), 287-291.
Zhang G., Yang Z. \& Dong S., 2011. Interspecific competitiveness affects the total biomass yield in an alfalfa and corn intercropping system. Field Crops Res., 124(1), 66-73.

Zoro Bi I.A., Koffi K.K. \& Djè Y., 2003. Caractérisation botanique et agronomique de trois espèces de cucurbites consommées en sauce en Afrique de l'Ouest: Citrullus sp., Cucumeropsis mannii Naudin et Lagenaria siceraria (Molina) Standl. Biotechnol. Agron. Soc. Environ., 7(34), 189-199.

Zuofa K., Tariah N.M. \& Isirimah N.O., 1992. Effects of groundnut, cowpea and melon on weed control and yields of intercropped cassava and maize. Field Crops Res., 28, 309-314.

(51 réf.) 